\title{
The Study of Ceramic Friction and Flow Stress on Wavelet Neural Network
}

\author{
Zhanjun Liu \\ Faculty of Aerospace Engineering, Shenyang Aerospace University, Shenyang, China
}

\begin{abstract}
The interface friction is used to evaluate the plastic flow behavior of workpieces material in different conditions such as temperature, pressure, strain rate and strain distribution. It is analyzed that deformation and material flow by the ring compression method during compression, which is contained with the theoretical calculation of different interface friction ring inside diameter and the height of the change. The synthetical symptoms of incremental method is concluded. Using wavelet and mixed data merge does the intelligence diagnosis to the defect of incremental method, which is integrated with data , characteristic, decision grate and nerve network. A model of wavelet neural network is constructed. In order to reduce defect analysis, the excellent diagnosis way is studied with the information of many sources fill and redundant. The result is given that using mixed data merge may raise tolerate character with the help of many sources fill and redundant, and using wavelet and mixed data merge does the effective diagnosis of ceramic friction and flow stress.
\end{abstract}

Keywords-wavelet neural; network; defect; incremental method; friction factor

\section{INTRODUCTION}

In plastic processing and forming process, lubricant is reasonably used to avoid the direct contact between mold and deformation of materials effectively, reduce the friction resistance and residual stress, reduce the inhomogeneity of workpiece deformation, to improve the surface quality, and prolong the service life of mould, and improve labor productivity. It is very important to evaluate the interface friction between die and workpiece, and it is analyzed to lubricant in the friction and lubrication behaviors under various working conditions, such as temperature, pressure, strain rate and working time on the influence of the lubricant used. In addition, the evaluation of interface friction is effective understanding of the workpiece material in different conditions such as temperature, pressure, strain rate and strain distribution under the condition of plastic flow behavior. Friction factor or friction coefficient is the measure of friction parameters in material forming, the most important used commonly is the ring compression method [1] [3]. It is a kind method of determining boundary in the plastic forming and friction behavior of the traditional method, through compared with other methods, it is also considered to determine material flow stress of feasible and effective method.

Precision plastic forming is that near net forming parts, as much as possible to reduce defects, lower material and mould cost. For the plastic itself is poorer, ceramic material is extremely difficult machining, in order to realize the near net forming of part, it is crucial to make full use of superplasticity.
At the same time, the ceramic material of high temperature and high pressure is molding process of forming dies and the selection of lubricant, it also put forward very high requirements, so it becomes the important basis for the ceramic material of molding that finding the right lubricant, exploring the use of lubricant characteristics, evaluation of ceramic under the condition of material flow and forming performance[4].

\section{THE BASIC THEORY OF INFORMATION FUSION TECHNOLOGY}

Information fusion or data fusion is to test the data from multiple sources, correlation, estimation and comprehensive grade, various processing, in order to obtain the accurate estimate of the state of the object to be tested and evaluated. The advantage of the coordination and joint action is by using multi-sensor to enhance the overall performance of the detection system[5].

Information fusion system is often will divide the data fusion layer, feature fusion layer and decision-making fusion of three levels. Policy makers refers to the fusion before integration, each sensor signal of local processing is done first, namely each sensor and the corresponding processing unit are respectively independently completed the feature extraction and decision tasks, such as correlation is be into the fusion center. Therefore, the essence of this method is based on certain criteria and the credibility of each decision makes optimal decisions. Policy makers fusion is a senior of data fusion fusion. Its advantage is small amount of data communication, real-time performance is good, which can handle asynchronous information fusion of different types of information effectively. In one or several sensor failure, the system can still continue to work, which have good fault tolerance, the reliability of the system is high. Therefore, policy makers fusion is a hotspot in the research of the information fusion. However, this technology also has its disadvantages, such as the loss of the original information, the time varying characteristics of the object and prior knowledge acquisition are difficult, the knowledge base of features are such as huge amount and difficult to get the application[6].

In the actual choice, blend mode should be determined by the specific problems. Fault diagnosis is in dynamic sealing lip seal shaft, due to the nature of a variety of testing equipment, for all kinds of detection methods of defect information model is different, it is difficult to directly related to its, so it is not easy for data fusion. By the testing equipment defect features of inconsistency makes using feature layer convergence difficulties, therefore, more practical choice is to fusion decision makers[7]. This way is not only minimal output 
information constraints on testing equipment, and system reliability is higher.

\section{The Structure of THE WAVELEt NeURAL Network MODEL}

Artificial neural network model have a hopfield net, forward multi-layer neural network and adaptive resonance theory, such as a variety of types model. Most applications in the field is of diagnosis, the most effective is the forward multilayer neural network model. Because the network model is used in the process of learning training after the error propagation algorithm, it is also called the BP network. BP network is all neurons within the layers of adjacent layer, neurons unavailability of bonding is strength value. Weight by known is to input and output information of the training sample, to adjust the network training and learning. This kind of adjustment is by BP algorithm. BP algorithm is a kind of error correction model learning algorithm, which is composed of forward and reverse transmission process[8]. In the forward propagation, the input information is from the input layer, through the role function, which is step by step to the hidden layer and output layer, if it can not get the desired output in the layer, then it came into the back propagation process, the error information is connecting path along the original return, by modifying the weights between neurons in each layer, make the output error is minimum. M input mapping of wavelet network model is as follows :

$$
f(X)=\sum_{i=1}^{n} W_{i} \boldsymbol{\Phi}_{i}\left(\frac{X-b_{i}}{a_{i}}\right)
$$

The wavelet basis function network is to select Morlet wavelet. The form of wavelet for:

$$
\psi(x)=e^{-\frac{x^{2}}{2}} \cos (5 x)
$$

The corresponding discrete form as follows:

$$
\Phi_{m n}(x)=a_{0}^{-m / 2} \Phi\left(a_{0}^{-m} x-n b_{0}\right)
$$

With wavelet basis function, it also needs through timefrequency analysis to determine the $\mathrm{m}, \mathrm{n}$. Setting can be used to support.

$$
\left\|f_{t}\right\|^{2}=\frac{1}{P} \sum_{i=1}^{P} y_{i}^{2} \bullet \Delta_{X_{i}},\left(\Delta X_{i}=X_{i}-X_{i-1}\right)
$$

Set energy is estimated to be in, there are:

$$
Q_{\varepsilon}^{+}(f)=\left[X_{\min }, X_{\max }\right] \bullet\left[\omega_{\min }^{*}, \omega_{\max }^{*}\right]
$$

By the above all kinds of available and estimate:

$$
\begin{aligned}
& n_{\text {max }}=\operatorname{int}\left\{-\frac{X_{\max } \bullet a^{-m}}{b}\right\} \\
& n_{\text {min }}=-\operatorname{int}\left\{-\frac{X_{\text {min }} \bullet a^{-m}}{b}\right\}
\end{aligned}
$$

With time and frequency region, it can determine the wavelet base. So called determined wave is to determine the wavelet base vector $\Phi$, and values range. By these results is given in literature, for each identified is values range.

\section{The Structure of the WaVelet Neural Network LEARNING ALGORITHM}

On the basis of the wavelet neural network structure, structure of wavelet neural network is learning algorithm. Because wavelet networks are of feedforward network, it can be the gradient descent method.

Network input for: $X=(x 1, x 2, x 3, \ldots \ldots ., x n)$, the first $k$ error of the sample is set as follows:

$$
f(X)=\sum_{i=1}^{m} w_{i} \phi_{i}\left(\frac{X-b_{i}}{a_{i}}\right)
$$

$$
E_{k}=\frac{1}{2}\left[\sum_{j=1}^{n}\left(y_{j}^{k}-f_{j}^{k}\right)^{2}\right]
$$

$$
(\mathrm{k}=1,2 \ldots \ldots, \mathrm{p})
$$

The expectations is for the first $\mathrm{k}$ sample. The error sum of squares is all input samples, it is as follows:

$$
E=\sum_{k} E_{k}=\frac{1}{2}\left[\sum_{j=1}^{n}\left(y_{j}^{k}-f_{j}^{k}\right)^{2}\right]
$$

$$
\Delta_{W_{i}}=-a \frac{\partial E_{k}}{\partial W_{i}}
$$




$$
\Delta_{W_{i}}=-a \frac{\partial \sum E}{\partial W_{i}}=a \sum_{k}\left(y^{k}-f_{i}^{k}\right)
$$

According to the rules for the minimum mean square error, $\mathrm{w}$ should change in the direction, it is shown by the negative partial, derivatives is proportional to the error. Due to the correct w should make the error sum of squares of all patterns, to the minimum, it should be proportional to the sum of all patterns of negative gradient, after a sufficient number of training, it can make the network value w, eventually memory is under their internal relations[9].

In the electromagnetic forming process, most of the deformation is in pulse pressure disappears. Under the effect of pulse pressure, the metal is obtained great acceleration, and got a lot of kinetic energy, metal forming is mainly depended on the kinetic energy to achieve. The electromagnetic forming is with pure electromagnetic properties, its forming process is not affected by mechanical inertia of the moving parts, so the electromagnetic forming can achieve rapid processing, which can work hundreds of times per minute. And the intensity of magnetic pulse can be accurately controlled, which is easy to realize mechanization and automation. The magnetic field can penetrate the nonconductive material, so to have a non-metallic coating or in forming the part with container. The electromagnetic forming is medium pressure on the workpiece with magnetic field, which is no mechanical contact. So the workpiece does not produce friction, which is no lubrication. The electromagnetic forming is a single-mode forming, which simplified the mould manufacturing, to increased the processing flexibility. The electromagnetic forming parts are with high precision, residual stress low, and the springback is small. The process of materials are best used in below $0.15 \mathrm{um}$, the high resistance materials need to be adopt the high frequency equipment, the driven piece of structure is used on the tooling. Electromagnetic forming is often used in flat workpieces and the forming of tube blank.

\section{The Standard Calibration CURVE of Ring COMPRESSION METHOD}

In the process of compression method measuring the interface friction, it should be the premise of accurate calibration curve. Not all circle geometry size is fit for the ring compression method, considering the circle on the application of the sensitivity of the experimental results and practical convenience, $6: 3: 2$ size is widely used. According to the theory analysis, the 6:3:2 size of ring friction factor standard calibration curve as shown in Fig.1, it can see, in the actual deformation measure circle and under the condition of inner diameter reduction, the actual interface friction factor can be marked on the diagram[10].

Ring compression method is the effective method to predict material flow stress. According to theoretical analysis, it can be calculated relative to the average stress, so it can draw calibration curve of changing with ring height under the condition of different friction factors. The average stress of ring standard calibration curve of 6:3:2 size is shown in Fig.2, it can see, if the friction factor is measured, the relative average stress can be obtained according to the practical circle deformation, and then it can calculate the average normal stress, according to the actual load stress, it can be concluded that the actual flow stress of material.

The polished ring specimen is placed in a open plane compression mold, mould and upper and lower blocks adopt high strength graphite, at the same time, the surface of the blocks is polished to the same roughness with sample. Furnace charging will be configured before spray lubricant evenly over the surface of the specimen and briquetting, the thickness is about $0.2 \mathrm{~mm}$.

The tube billet is bulging, and working coil is in the tube, when the pulse current is through the coil, a magnetic pressure pushs the tube outside billet mold. This method can be used for bulging and connection technology. Reducing is tube billet. Within the workpiece, the coil is inside the magnetic pressure to the workpiece of the compression modulus, which is often used for forming and connection process[5]. The flat blank is forming. A spiral flat coil is placed on a flat blank, when the pulse current is through the coil, the electromagnetic puts parts into the mould. This method can be used for flat blank forming, finishing and cutting. Electromagnetic forming is very suitable for tube and pipe, tube and plate connection technology may realize the connection of tube and the core shaft[6].

It is diversity that components of complex mechanical system failure mode and failure mechanism, failure prediction time is also very complex. In the process of the development of the system, with trials, in view of the different failure modes, improvement measures will be taken, and the reliability of the different failure modes may correspond to different growth patterns. But traditional reliability growth model, such as Duane model[7], the AMSAA model, usually only single reliability growth model is set up, which only discuss the time system failure and the failure number. the relationship between the considering the internal system corresponding to different failure mechanism is the effects of different improvement measures on the system reliability growth. This is a matter of engineering who has long been recognized, but because of the hybrid model parameter estimation problem is difficult, and thus has not an in-depth study. In this paper, through the establishment of a hybrid reliability growth model[8], it can reflect the different failure mechanism on the system reliability growth impact of different. To contain two kinds of failure mechanism of mixed AMSAA model is as an example in this paper, that is the main theory of this method.

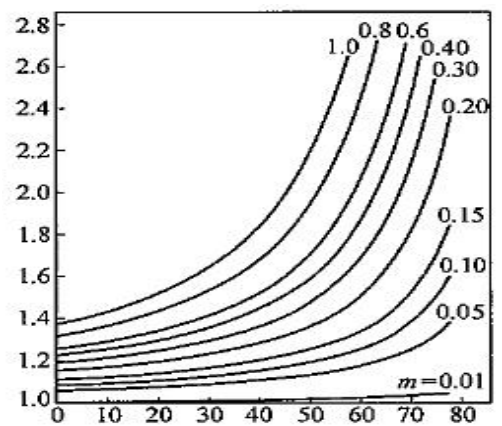

FIGURE I. FRICTION FACTOR STANDARD CALIBRATION CURVE 


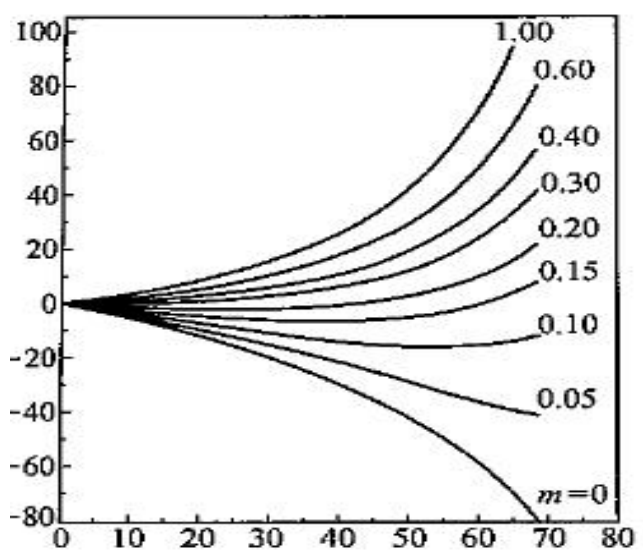

FIGURE II. THE AVERAGE STRESS OF RING CALIBRATION CURVE

\section{CONCLUSIONS}

The interface friction is used to evaluate the plastic flow behavior of workpiece material in different conditions such as temperature, pressure, strain rate and strain distribution. It is analyzed that deformation and material flow by the ring compression method during compression, which is contained with the theoretical calculation of different interface friction ring inside diameter and the height of the change. The synthetical symptoms of incremental method is concluded. Using wavelet and mixed data merge does the intelligence diagnosis to the defect of incremental method, which is integrated with data, characteristic, decision grate and nerve network . A model of wavelet neural network is constructed. In order to reduce defect analysis, the excellent diagnosis way is studied with the information of many sources fill and redundant The result is given that using mixed data merge may raise tolerate character with the help of many sources fill and redundant, and using wavelet and mixed data merge does the effective diagnosis of ceramic friction and flow stress.

\section{REFERENCES}

[1] ScheyJA.Monogr and Textbooks in Mater .Sci.1.Metal deformation process, friction and lubrication[M].New York:Marcel Dekker Lnc, p807-815,2010.

[2] Male.AT,Cockroft M G.A method for the determination of the coefficient of friction of metals under conditions of bulk plastic deformation[J].J.Inst.Met, p 38-46, 2004.

[3] R Kolleck et al, Hot Forming and Cold Forming Two Complementary Processes for Lightweight Auto Bodies, in proceedings from The International Conference New Development in Sheet Metal Forming Technology, Stuttgart, Germany, p.235-244, 2004.

[4] Guofang, Reliability Data Collection And Analysis, National Defence Industry Press, p22,1995.

[5] Middleman. S, An introduction to fluid dynamics, John Wiley and Sons Inc,pp.24-25,1998.

[6] Gutowski T .G, Advanced composites manufacturing, John Wiley \& Sons Inc, New York, pp. 416-429, 1997.

[7] Rudd C D, Long A C, Kendall K N, Mangin C G E. Liquid molding technologies, Woodhead Publishing Ltd, London,pp.203-253, 1997.

[8] White Frank. M, Fluid mechanics (third edition), McGraw-Hill Inc, Highstown,pp.198-222, 1994.

[9] Wen.WeiDong, Prediction model based on the fuzzy reliability, aircraft engine, Beijing, pp.322-323,2003.
[10] Liu.ZhanJun, Difficulty reliability prediction research of titanium plate stamping forming based on the fuzzy control, plastic engineering journal, Beijing,pp. 63-66,2005 (6) . 